$11-1-2012$

\title{
Modified EDF Goodness of Fit Tests for Logistic Distribution under SRS and RSS
}

\author{
S. A. Al-Subh \\ Jerash Private University, Jerash, Jordan \\ M. T. Alodat \\ Qatar University \\ Kamaruzaman Ibrahim \\ University Kebangsaan Malaysia \\ Abdul Aziz Jemain \\ Kebangsaan University, Selangor, Malaysia, azizj@pkrisc.cc.ukm.my
}

Follow this and additional works at: http://digitalcommons.wayne.edu/jmasm

Part of the Applied Statistics Commons, Social and Behavioral Sciences Commons, and the Statistical Theory Commons

\section{Recommended Citation}

Al-Subh, S. A.; Alodat, M. T.; Ibrahim, Kamaruzaman; and Jemain, Abdul Aziz (2012) "Modified EDF Goodness of Fit Tests for Logistic Distribution under SRS and RSS," Journal of Modern Applied Statistical Methods: Vol. 11 : Iss. 2 , Article 11.

DOI: $10.22237 /$ jmasm/1351743000

Available at: http://digitalcommons.wayne.edu/jmasm/vol11/iss2/11 


\title{
Modified EDF Goodness of Fit Tests for Logistic Distribution under SRS and RSS
}

\author{
S. A. Al-Subh \\ M. T. Alodat \\ Kamaruzaman Ibrahim Abdul Aziz Jemain \\ Jerash Private University, \\ Jerash, Jordan \\ Qatar University, \\ Qatar \\ Kebangsaan University, \\ Selangor, Malaysia
}

Modified forms of goodness of fit tests are presented for the logistic distribution using statistics based on the empirical distribution function (EDF). A method to improve the power of the modified EDF goodness of fit tests is introduced based on Ranked Set sampling (RSS). Data are collected via the Ranked Set Sampling (RSS) technique (McIntyre, 1952). Critical values for the logistic distribution with unknown parameters are provided and the powers of the tests are given for a number of alternative distributions. A simulation study is presented to illustrate the power of the new method.

Key words: Goodness of fit tests, empirical distribution function, power, logistic distribution, ranked set sample, Kolmogorov-Smirnov statistic.

Introduction

Many sampling methods can be used to estimate the population parameters. However, in many situations the experimental units for the variable of interest can be more easily ranked than quantified. The use of the method of ranked set sampling (RSS) in these situations is highly beneficial and is superior to simple random sampling (SRS). In many agricultural and environmental studies, it is possible to rank the experimental or sampling units with respect to the variable of interest, without actually measuring them; this usually results in costsavings. The RSS sampling method can be used when measurements of sample units, drawn from the population of interest, are very

Sameer Al-Subh is an Assistant Professor in the Department of Mathematics. Email him at: salsubh@yahoo.com. M. T. Alodat is an Associate Professor in the Department of Mathematics, Statistics and Physics, Qatar University, Qatar. Email him at: alodatmts@yahoo.com. Kamaruzaman Ibrahim is a Professor of statistics in the School of Mathematical Sciences. Email him at: kamarulz@ukm.my. Abdul Aziz Bin Jemain is a Professor of statistics in the School of Mathematical Sciences. Email him at: azizj@ukm.my. laborious or costly in time or money, but can be easily arranged (ranked) in order of their magnitude.

McIntyre (1952) was the first to introduce ranked set sampling (RSS). RSS gives a sample that is more informative than a simple random sample (SRS) concerning a population of interest. The RSS technique can be described as follows: Select $m$ random samples from a population of interest each of size $m$. From the $i^{\text {th }}$ sample use a visual inspection to detect the $i^{\text {th }}$ order statistic and choose it for actual quantification, for example, $Y_{i}, i=1, \ldots, m$. Assuming the ranking is perfect RSS is the set of the order statistics $Y_{1}, \ldots, Y_{m}$. The RSS technique can be repeated $\mathrm{r}$ times to obtain additional observations; these resulting measurements form an RSS of size $\mathrm{rm}$.

Two factors affect the efficiency of an RSS: set size and ranking errors. The larger the set size, the larger the efficiency of RSS, while the larger the set size the more the difficulty in the visual ranking and hence the larger the ranking error (Al-Saleh \& Al-Omari, 2002). Takahasi and Wakimoto (1968) provided the theoretical setups for RSS by showing that the mean of an RSS is the minimum variance unbiased estimator for a population mean. Dell and Clutter (1972) further showed that the sample mean RSS remains unbiased and more 
efficient than the sample mean even if ranking is imperfect.

Several authors have modified RSS to reduce the error in ranking and to make visual ranking tractable by experimenter. (For details about RSS and its modifications, see Muttlak, 1997; Samawi, et al, 1996; Al-Odat \& Al-Saleh, 2001; Bhoj, 1997; Chen, 2000; Patil, et al, 1994a).

Stockes and Sager (1988) studied the characterization of RSS. In addition, for deriving the null distribution of their proposed test, they introduced an unbiased estimator for the population distribution function based on the empirical distribution function of RSS. Also, proposed a Kolmogorov-Smirnov goodness of fit test based on the empirical distribution function (EDF). Ibrahim et al. (2011) introduced a method to improve the power of the Chisquare goodness of fit test based on RSS. They used Kullback-Leibler information to compare data collected via both SRS and RSS and conducted a simulation study for the power of Chi-square test of the new method.

Al-Subh et al. (2009) conducted a comparison study for the power of a set of EDF goodness of fit tests for the logistic distribution under SRS and RSS. This article proposes a method to improve the power of the EDF goodness of fit tests for logistic distribution under RSS and uses a simulation study to compare the powers of each test under the RSS.

\section{MEDF Goodness of Fit Tests}

Stephens (1974) presented a practical guide to goodness of fit tests using statistics based on the EDF. Green and Hegazy (1976) studied modified forms of the KolmogorovSmirnov $D$, Cramer-von Mises $W^{2}$ and Anderson-Darling $A^{2}$ goodness of fit tests. Stephens (1979) gave goodness of fit tests for the logistic distribution based on a SRS; a comprehensive survey of goodness of fit tests based on SRS can be found in Stephens (1986).

Let $X_{1}, X_{2}, \ldots, X_{n}$ be a random sample from the distribution function $F(x)$ where $X_{1}<X_{2}, \ldots,<X_{n}$ is the order statistics of random sample of size $n$ from $F(x)$. Assume that the objective is to test the statistical hypotheses

$$
\begin{gathered}
H_{o}: F(x)=F_{o}(x) \forall x \\
\text { vs. } \\
H_{1}: F(x) \neq F_{o}(x)
\end{gathered}
$$

for some $x$, where $F_{o}(x)$ is a known distribution function.

The MEDF goodness of fit tests in SRS are defined as:

a) Tests related to Kolmogorov statistic, $D$ :

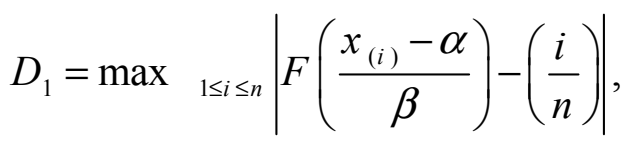

where $i=1,2, \ldots, n$ and $n$ is the sample size.

$$
\begin{gathered}
D_{11}=\max _{1 \leq i \leq n}\left|F\left(\frac{x_{(i)}-\alpha}{\beta}\right)-\left(\frac{i}{n+1}\right)\right|, \\
D_{2}=\sum_{i=1}^{n}\left|F\left(\frac{x_{(i)}-\alpha}{\beta}\right)-\left(\frac{i}{n}\right)\right|, \\
D_{22}=\sum_{i=1}^{n}\left|F\left(\frac{x_{(i)}-\alpha}{\beta}\right)-\left(\frac{i+0.5}{n+1}\right)\right| \\
D_{3}=\sup _{1 \leq i \leq n}\left|F\left(\frac{x_{(i)}-\alpha}{\beta}\right)-\left(\frac{i}{n}\right)\right|,
\end{gathered}
$$

and

$$
\begin{aligned}
& D_{4}= \\
& \sum_{i=1}^{n} \max \left\{\left|\frac{i}{n}-F\left(\frac{x_{(i)}-\alpha}{\beta}\right)\right|,\left|\frac{i-1}{n}-F\left(\frac{x_{(i)}-\alpha}{\beta}\right)\right|\right\} .
\end{aligned}
$$


b) Tests related to Cramer-von Mises statistic, $W^{2}$ :

$$
\begin{gathered}
W_{o}=\sum_{i=1}^{n}\left[F\left(\frac{x_{(i)}-\alpha}{\beta}\right)-\left(\frac{2 i-1}{2 n}\right)\right]^{2}, \\
W_{11}=\sum_{i=1}^{n}\left[F\left(\frac{x_{(i)}-\alpha}{\beta}\right)-\frac{i}{n+1}\right]^{2}, \\
W_{21}=\sum_{i=1}^{n}\left[F\left(\frac{x_{(i)}-\alpha}{\beta}\right)-\left(\frac{2 i-1}{2(n+1)}\right)\right]^{2} .
\end{gathered}
$$

c) Tests related to Anderson-Darling statistic, $A^{2}$ :

$$
a a_{21}=
$$

$$
\begin{aligned}
-n-\frac{n}{(r+1)^{2}}\left\{\sum_{i=1}^{n}\left[\begin{array}{l}
(2 i-1) \ln F\left(\frac{x_{(i)}-\alpha}{\beta}\right) \\
\left.+(2 i+1) \ln \left(1-F\left(\frac{x_{(n-i+1)}-\alpha}{\beta}\right)\right)\right)
\end{array}\right]\right. \\
\left.-\left[\begin{array}{l}
(2 n+1) \ln F\left(\frac{x_{(n)}-\alpha}{\beta}\right) \\
-\ln \left(1-F\left(\frac{x_{(n-i+1)}-\alpha}{\beta}\right)\right)
\end{array}\right]\right\},
\end{aligned}
$$

$$
\begin{aligned}
& a a_{22}= \\
& -n-\left(\frac{2 n}{(n+1)^{2}}\right)\left[\left\{\sum_{i=1}^{n} i\left[\ln F\left(\frac{x_{(i)}-\alpha}{\beta}\right)\right]+\ln \left(1-F\left(\frac{x_{(n-i+1)}-\alpha}{\beta}\right)\right)\right]\right\} \\
& -\left(\frac{n}{(n+1)^{2}}\right)\left[0 . 2 5 \left\{\ln F\left(\frac{x_{(i)}-\alpha}{\beta}\right)\right.\right. \\
& \left.+\ln \left(1-F\left(\frac{x_{(n)}-\alpha}{\beta}\right)\right)\right\} \\
& +(n+0.75)\left\{\ln F\left(\frac{x_{(i)}-\alpha}{\beta}\right)\right. \\
& \left.\left.+\ln \left(1-F\left(\frac{x_{(1)}-\alpha}{\beta}\right)\right)\right\}\right]
\end{aligned}
$$

and

$$
\begin{aligned}
& a a_{12}= \\
& -(n+1)-\left(\frac{1}{n+1}\right)\left\{\sum_{i=1}^{n} 2 i\left[\begin{array}{l}
\ln F\left(\frac{x_{(i)}-\alpha}{\beta}\right) \\
+\ln \left(1-F\left(\frac{x_{(n-i+1)}-\alpha}{\beta}\right)\right)
\end{array}\right]\right\} .
\end{aligned}
$$

This study examines the case $F_{o}(x)=\left(1+e^{-(x-\alpha) / \beta}\right)^{-1}$, that is, for the logistic distribution. A simulation study is conducted to show that the test $T^{*}$ is more powerful than the test $T$ when compared based on samples of the same size. The power of the $T^{*}$ test can be calculated according to the equation:

$$
T^{*}(H)=P_{H}\left(T^{*}>d_{\alpha}\right),
$$

where $H$ is a cdf under alternative hypothesis $H_{1}^{*}$. Here $d_{\alpha}$ is the $100 \alpha$ percentage point of the distribution of $T^{*}$ and $H_{o}$. Due to the behavior of RSS test statistics relative to SRS 
test statistics, the efficiency of the test statistics is calculated as a ratio of powers:

$$
\text { eff }\left(T^{*}, T\right)=\frac{\text { power of } T^{*}}{\text { power of } T}
$$

where $T^{*}$ is more powerful than $T$ if eff $\left(T^{*}, T\right)>1$.

Test for Logistic Distribution

Let $X_{(1) 1}, X_{(2) 1}, \ldots, X_{(m) r}, n=m r$ be a RSS of size $n=m r$ from a distribution function $F(x)$. The test described is an uppertail test. A goodness of fit test is performed for the hypotheses:

$$
\begin{gathered}
H_{o}: F(x)=F_{o}(x) \forall x, \\
\text { vs. } \\
H_{1}: F(x) \neq F_{o}(x)
\end{gathered}
$$

where $F_{o}(x)=\left(1+e^{-(x-\alpha) / \beta}\right)^{-1}$.

If $\alpha$ and $\beta$ are unknown, then they may be estimated using their maximum likelihood estimator i.e, from $l(\alpha, \beta)$, by making the log likelihood function of the data:

$l(\alpha, \beta)=-n \ln (\beta)-\sum_{i=1}^{n}\left(z_{i}\right)-2 \sum_{i=1}^{n} \ln \left(1+e^{-z_{i}}\right)$,

and in RSS by

$$
\begin{aligned}
l(\alpha, \beta)= & -n \ln (\beta)+\sum_{j=1}^{r} \sum_{i=1}^{m}(i-1) \ln F\left(z_{(i) j}\right) \\
& +\sum_{j=1}^{r} \sum_{i=1}^{m}(m-i) \ln \left(1-F\left(z_{(i) j}\right)\right) \\
& +\sum_{j=1}^{r} \sum_{i=1}^{m} \ln f\left(z_{(i) j}\right),
\end{aligned}
$$

where

$$
z_{i}=\left(x_{i}-\alpha\right) / \beta, z_{(i) j}=\left(x_{(i) j}-\alpha\right) / \beta
$$

and

$$
f\left(z_{(i) j}\right)=\frac{e^{-z_{(i) j}}}{\beta\left(1+e^{-z_{(i) j}}\right)^{2}} .
$$

Using the tests given in (1) and based on the data $X_{(1) 1}, X_{(2) 1}, \ldots, X_{(m) r}, n=m r$ called via the RSS.

\section{Power Comparison Algorithm}

Let $T$ denote a test in (1) based on SRS and $T^{*}$ be the same test, but based on RSS. To compare the power of the test $T^{*}$ with the power of the test $T$ based on samples of the same size, first the algorithm to calculate the percentage points is introduced:

1. Let $x_{(i) j}$ be a random sample from $F_{o}(x)$.

2. Estimate parameters $\alpha$ and $\beta$ from the sample by maximum likelihood; the estimates are given by (3).

3. Find the $\operatorname{EDF} F_{n}^{*}(x)$ as follows:

$$
\begin{aligned}
& F_{n}^{*}(x)=\frac{1}{m r} \sum_{j=1}^{r} \sum_{i=1}^{m} I\left(x_{(i) j} \leq x\right), \\
& I\left(x_{(i) j} \leq x\right)=\left\{\begin{array}{l}
1, \mathrm{x}_{(i) j} \leq x, \\
0, \text { o.w. }
\end{array}\right.
\end{aligned}
$$

4. Use $F_{n}^{*}(x)$ to calculate the value of $T^{*}$ as in (1).

5. Repeat steps one through four 10,000 times to obtain $T_{1}^{*}, \ldots, T_{10,000}^{*}$.

6. The percentage point $d_{\alpha}$ of $T^{*}$ is approximated by the $(1-\alpha) 100$ quantile of $T_{1}^{*}, \ldots, T_{10,000}^{*}$. 
The following algorithm is designed to obtain the power of $T^{*}$ at a distribution, for example, $H$, under $H_{o}$ :

1. Let $x_{(i) j}$ be a random sample from $F_{o}(x)$.

2. Estimate the parameters $\alpha, \beta$ from the sample by maximum likelihood; the estimates are given by (3).

3. Find the EDF $F_{n}^{*}(x)$ as in (4).

4. Calculate the value of $T^{*}$ in (1).

5. Repeat steps one through four 10,000 times to obtain $T_{1}^{*}, \ldots, T_{10,000}^{*}$.

6. Calculate the power of

$$
T^{*}(H) \approx \frac{1}{10,000} \sum_{t=1}^{10,000} I\left(T_{t}^{*}>d_{\alpha}\right),
$$

where $I($.$) stands for indicator function.$

\section{Results}

A simulation study was conducted to compare the power of $T$ and $T^{*}$. The power, as well as the percentage point, of each test are approximated based on a Monte Carlo simulation of 10,000 iterations according to the algorithm described previously. Table 1 shows the percentage points for the $5 \%$ level for the null hypotheses of the logistic distribution for RSS. The efficiency of the tests was compared for different sized samples: $r=3,5,10,25$; different set sizes: $m=2,3$; and different alternative distributions: Normal $=N\left(\alpha, \beta^{2}\right)$, Laplace $=L(\alpha, \beta), \quad$ Cauchy $=C(\alpha, \beta)$, StudentT $=S(5), \quad$ Uniform $=U(\alpha, \beta)$, and Lognormal $=\operatorname{LN}(\alpha, \beta)$. Comparisons were made only for cases where the data are quantified via RSS. Simulation results are shown in the Tables 2 and 3. For the lognormal and uniform distributions, computations show that the powers of all test statistics equal one, thus, these powers are not reported.

Based on study results, the following conclusions are put forth:

1. The efficiencies in Tables 1 and 3 are all greater than 1; this indicates that the MEDF tests under ERSS are more powerful than their counterparts in SRS.

2. Tables 1-3 show that the efficiency increases as the distribution under the alternative hypothesis departs to asymmetry.

3. Power increases as the sample size $n$ increases.

4. Power is equal to one for the lognormal and uniform distributions.

5. The MEDF tests based on data collected via RSS are more powerful than the EDF tests based on an SRS of the same size.

\section{Conclusion}

The power of a set of modified EDF goodness of fit tests was shown to be improved if a sample is collected via the RSS method, as opposed to the SRS method. Moreover, modified EDF tests show excellent power performance in comparison to their SRS counterparts. Although this study is limited to the logistic distribution under the null hypothesis, it could be easily extended to other distributions.

\section{References}

Al-Odat, M. T., \& Al-Saleh, M. F. (2001). A variation of ranked set sampling. Journal of Applied Statistical Science, 10, 137146.

Al-Saleh, M. F., \& Al-Omari, A. I. (2002). Multistage ranked set sampling. Journal of Statistical planning and Inference, 102, 273286.

Ibrahim, K., Alodat, M. T., Jemain, A. A., \& Al-Subh, S. A. (2011) Chi-square test for goodness of fit for logistic distribution using ranked set sampling and simple random sampling. Statistica \& Applicazioni, IX(2), 111128. 
EDF GOODNESS OF FIT TESTS FOR LOGISTIC DISTRIBUTION UNDER SRS AND RSS

Table 1: Percentage Points for SRS and RSS, $\alpha=0.05$

\begin{tabular}{|c|c|c|c|c|c|c|c|c|c|c|}
\hline \multirow{3}{*}{ Test } & \multicolumn{5}{|c|}{ SRS } & \multicolumn{5}{|c|}{ RSS } \\
\hline & \multicolumn{5}{|c|}{$n$} & \multicolumn{5}{|c|}{$n$} \\
\hline & 6 & 10 & 20 & 30 & 50 & 6 & 10 & 20 & 30 & 50 \\
\hline$D_{1}$ & 0.269 & 0.215 & 0.158 & 0.132 & 0.103 & 0.271 & 0.221 & 0.166 & 0.140 & 0.116 \\
\hline$D_{11}$ & 0.198 & 0.175 & 0.141 & 0.122 & 0.099 & 0.200 & 0.175 & 0.143 & 0.126 & 0.107 \\
\hline$D_{2}$ & 0.723 & 0.885 & 1.191 & 1.460 & 1.861 & 0.747 & 0.932 & 1.312 & 1.631 & 2.249 \\
\hline$D_{22}$ & 0.693 & 0.881 & 1.201 & 1.470 & 1.867 & 0.712 & 0.884 & 1.244 & 1.537 & 2.128 \\
\hline$D_{3}$ & 0.287 & 0.228 & 0.168 & 0.140 & 0.110 & 0.291 & 0.237 & 0.177 & 0.150 & 0.122 \\
\hline$D_{4}$ & 1.096 & 1.283 & 1.618 & 1.903 & 2.311 & 1.115 & 1.322 & 1.731 & 2.062 & 2.694 \\
\hline$W W_{0}$ & 0.081 & 0.087 & 0.091 & 0.097 & 0.096 & 0.087 & 0.098 & 0.109 & 0.118 & 0.137 \\
\hline$W W_{11}$ & 0.085 & 0.089 & 0.093 & 0.098 & 0.096 & 0.088 & 0.089 & 0.098 & 0.104 & 0.122 \\
\hline$W W_{21}$ & 0.116 & 0.110 & 0.104 & 0.105 & 0.101 & 0.118 & .110 & 0.109 & 0.112 & 0.127 \\
\hline$a a_{21}$ & 0.261 & 0.376 & 0.488 & 0.550 & 0.593 & 0.312 & 0.393 & 0.499 & 0.549 & 0.690 \\
\hline$a a_{22}$ & 0.745 & 0.757 & 0.726 & 0.727 & 0.711 & 0.761 & 0.754 & 0.740 & 0.751 & 0.850 \\
\hline$a a_{12}$ & 0.421 & 0.513 & 0.577 & 0.618 & 0.641 & 0.460 & 0.505 & 0.573 & 0.623 & 0.762 \\
\hline
\end{tabular}


Table 2: Efficiency Values of Tests Using RSS with respect to SRS for $n=6,10,20,30,50$ and $\alpha=0.05$

\begin{tabular}{|c|c|c|c|c|c|c|}
\hline \multirow{2}{*}{$H$} & \multirow{2}{*}{$T$} & \multicolumn{5}{|c|}{$n$} \\
\hline & & 6 & 10 & 20 & 30 & 50 \\
\hline \multirow{12}{*}{$N\left(\theta, \sigma^{2}\right)$} & $D_{1}$ & 5.52 & 1.55 & 1.16 & 1.01 & 1 \\
\hline & $D_{11}$ & 0.27 & 1.98 & 1.49 & 1.06 & 1 \\
\hline & $D_{2}$ & 8.67 & 1.21 & 0.98 & 1 & 1 \\
\hline & $D_{22}$ & 0.36 & 2.05 & 1.11 & 1 & 1 \\
\hline & $D_{3}$ & 1.42 & 1.39 & 1.08 & 0.99 & 1 \\
\hline & $D_{4}$ & 1.97 & 1.18 & 0.99 & 1 & 1 \\
\hline & $W W_{0}$ & 1.95 & 1.34 & 0.99 & 1 & 1 \\
\hline & $W W_{11}$ & 1.54 & 2.29 & 0.94 & 1 & 1 \\
\hline & $W W_{21}$ & 1.25 & 2.17 & 0.94 & 1 & 1 \\
\hline & $a a_{21}$ & 2.12 & 2.17 & 1 & 1 & 1 \\
\hline & $a a_{22}$ & 4.38 & 4.91 & 0.98 & 1 & 1 \\
\hline & $a a_{12}$ & 1 & 3.21 & 1.52 & 1 & 1 \\
\hline \multirow{12}{*}{$L(\theta, \sigma)$} & $D_{1}$ & 1.38 & 1.43 & 1.4 & 1.21 & 1.04 \\
\hline & $D_{11}$ & 1.19 & 1.38 & 1.42 & 1.24 & 1.05 \\
\hline & $D_{2}$ & 1.08 & 1.43 & 1.27 & 1.04 & 1 \\
\hline & $D_{22}$ & 0.85 & 1.35 & 1.7 & 1.31 & 1 \\
\hline & $D_{3}$ & 1.05 & 1.16 & 1.18 & 1.11 & 1.02 \\
\hline & $D_{4}$ & 1.51 & 1.65 & 1.26 & 1.02 & 1 \\
\hline & $W W_{0}$ & 1.26 & 1.4 & 1.17 & 1.03 & 1 \\
\hline & $W W_{11}$ & 0.83 & 1.51 & 1.6 & 1.21 & 1 \\
\hline & $W W_{21}$ & 0.51 & 0.94 & 1.47 & 1.21 & 1 \\
\hline & $a a_{21}$ & 1.15 & 1.66 & 2.11 & 1.52 & 1 \\
\hline & $a a_{22}$ & 0.82 & 1.48 & 2.17 & 1.53 & 1 \\
\hline & $a a_{12}$ & 0.47 & 0.95 & 2.19 & 1.57 & 1 \\
\hline
\end{tabular}


Table 2 (continued): Efficiency Values of Tests Using RSS with respect to SRS for $n=6,10,20,30,50$ and $\alpha=0.05$

\begin{tabular}{|c|c|c|c|c|c|c|}
\hline \multirow{2}{*}{$H$} & \multirow{2}{*}{$T$} & \multicolumn{5}{|c|}{$n$} \\
\hline & & 6 & 10 & 20 & 30 & 50 \\
\hline \multirow{12}{*}{$C(\theta, \sigma)$} & $D_{1}$ & 1.7 & 2.07 & 2.17 & 2.09 & 1.75 \\
\hline & $D_{11}$ & 0.72 & 0.91 & 1.14 & 1.26 & 1.27 \\
\hline & $D_{2}$ & 1.28 & 1.41 & 1.56 & 1.68 & 1.56 \\
\hline & $D_{22}$ & 1.19 & 1.41 & 1.52 & 1.62 & 1.5 \\
\hline & $D_{3}$ & 1.03 & 1.26 & 1.42 & 1.54 & 1.42 \\
\hline & $D_{4}$ & 0.68 & 0.9 & 1.09 & 1.29 & 1.32 \\
\hline & $W W_{0}$ & 0.76 & 0.98 & 1.16 & 1.37 & 1.37 \\
\hline & $W W_{11}$ & 0.71 & 0.93 & 1.14 & 1.32 & 1.3 \\
\hline & $W W_{21}$ & 0.49 & 0.47 & 0.66 & 0.91 & 1.11 \\
\hline & $a a_{21}$ & 0.85 & 0.91 & 1 & 1.1 & 1.25 \\
\hline & $a a_{22}$ & 0.89 & 0.96 & 1.05 & 1.13 & 1.25 \\
\hline & $a a_{12}$ & 0.86 & 0.95 & 1.07 & 1.12 & 1.26 \\
\hline \multirow{12}{*}{$S(5)$} & $D_{1}$ & 1.97 & 1.75 & 1.59 & 1.25 & 1.01 \\
\hline & $D_{11}$ & 2.91 & 2.48 & 2.12 & 1.43 & 1.03 \\
\hline & $D_{2}$ & 1.96 & 2.13 & 1.11 & 0.98 & 1 \\
\hline & $D_{22}$ & 1.15 & 2.7 & 2.57 & 1.1 & 1 \\
\hline & $D_{3}$ & 1.8 & 1.48 & 1.32 & 1.13 & 1 \\
\hline & $D_{4}$ & 2.53 & 2.35 & 1.06 & 0.99 & 1 \\
\hline & $W W_{0}$ & 2.2 & 2 & 1.12 & 0.98 & 1 \\
\hline & $W W_{11}$ & 1.64 & 3.46 & 2.35 & 1.1 & 1 \\
\hline & $W W_{21}$ & 0.74 & 2.2 & 2.26 & 1.11 & 1 \\
\hline & $a a_{21}$ & 2.34 & 3.37 & 2.7 & 1.02 & 1 \\
\hline & $a a_{22}$ & 1.16 & 3.02 & 3.32 & 1.07 & 1 \\
\hline & $a a_{12}$ & 0.75 & 1.45 & 4.03 & 1.48 & 1 \\
\hline
\end{tabular}


IBRAHIM, ALODAT, JEMAIN \& AL-SUBH

Table 3: $1,000 \times$ Power Values for SRS and RSS-Two Unknown Parameters, $\alpha=0.05$

\begin{tabular}{|c|c|c|c|c|c|c|c|c|c|c|c|}
\hline \multirow{3}{*}{$H$} & \multirow{3}{*}{ Test } & \multicolumn{5}{|c|}{ SRS } & \multicolumn{5}{|c|}{ RSS } \\
\hline & & \multicolumn{5}{|c|}{$n$} & \multicolumn{5}{|c|}{$n$} \\
\hline & & 6 & 10 & 20 & 30 & 50 & 6 & 10 & 20 & 30 & 50 \\
\hline \multirow{12}{*}{$N\left(\theta, \sigma^{2}\right)$} & $D_{1}$ & 135 & 306 & 750 & 976 & 1000 & 254 & 473 & 872 & 983 & 1000 \\
\hline & $D_{11}$ & 42 & 115 & 522 & 911 & 1000 & 52 & 228 & 780 & 970 & 1000 \\
\hline & $D_{2}$ & 184 & 492 & 998 & 1000 & 1000 & 260 & 595 & 1000 & 1000 & 1000 \\
\hline & $D_{22}$ & 29 & 132 & 822 & 1000 & 1000 & 47 & 271 & 916 & 1000 & 1000 \\
\hline & $D_{3}$ & 125 & 336 & 819 & 1000 & 1000 & 245 & 466 & 887 & 1000 & 1000 \\
\hline & $D_{4}$ & 172 & 579 & 1000 & 1000 & 1000 & 339 & 685 & 1000 & 1000 & 1000 \\
\hline & $W W_{0}$ & 165 & 494 & 1000 & 1000 & 1000 & 330 & 661 & 1000 & 1000 & 1000 \\
\hline & $W W_{11}$ & 28 & 129 & 807 & 1000 & 1000 & 43 & 296 & 941 & 1000 & 1000 \\
\hline & $W W_{21}$ & 33 & 134 & 810 & 1000 & 1000 & 45 & 291 & 940 & 1000 & 1000 \\
\hline & $a a_{21}$ & 108 & 253 & 1000 & 1000 & 1000 & 235 & 550 & 1000 & 1000 & 1000 \\
\hline & $a a_{22}$ & 8 & 56 & 1000 & 1000 & 1000 & 35 & 275 & 1000 & 1000 & 1000 \\
\hline & $a a_{12}$ & 1 & 19 & 560 & 1000 & 1000 & 1 & 61 & 907 & 1000 & 1000 \\
\hline \multirow{12}{*}{$L(\theta, \sigma)$} & $D_{1}$ & 192 & 295 & 519 & 721 & 938 & 281 & 434 & 729 & 876 & 980 \\
\hline & $D_{11}$ & 98 & 211 & 473 & 687 & 930 & 117 & 296 & 673 & 852 & 977 \\
\hline & $D_{2}$ & 280 & 317 & 642 & 918 & 1000 & 308 & 465 & 818 & 956 & 1000 \\
\hline & $D_{22}$ & 205 & 218 & 414 & 705 & 1000 & 182 & 295 & 703 & 921 & 1000 \\
\hline & $D_{3}$ & 281 & 394 & 632 & 800 & 963 & 314 & 469 & 744 & 888 & 982 \\
\hline & $D_{4}$ & 164 & 272 & 660 & 941 & 1000 & 252 & 448 & 831 & 964 & 1000 \\
\hline & $W W_{0}$ & 200 & 335 & 715 & 930 & 1000 & 269 & 475 & 838 & 962 & 1000 \\
\hline & $W W_{11}$ & 89 & 166 & 459 & 769 & 998 & 78 & 256 & 734 & 931 & 1000 \\
\hline & $W W_{21}$ & 165 & 214 & 447 & 747 & 997 & 90 & 201 & 658 & 907 & 1000 \\
\hline & $a a_{21}$ & 170 & 199 & 340 & 621 & 1000 & 217 & 331 & 718 & 941 & 1000 \\
\hline & $a a_{22}$ & 115 & 146 & 304 & 603 & 1000 & 101 & 216 & 660 & 921 & 1000 \\
\hline & $a a_{12}$ & 74 & 108 & 268 & 573 & 997 & 35 & 103 & 586 & 899 & 1000 \\
\hline
\end{tabular}


EDF GOODNESS OF FIT TESTS FOR LOGISTIC DISTRIBUTION UNDER SRS AND RSS

Table 3 (continued): 1,000 $\times$ Power Values for SRS and RSS-Two Unknown Parameters, $\alpha=0.05$

\begin{tabular}{|c|c|c|c|c|c|c|c|c|c|c|c|}
\hline \multirow{3}{*}{$H$} & \multirow{3}{*}{ Test } & \multicolumn{5}{|c|}{ SRS } & \multicolumn{5}{|c|}{ RSS } \\
\hline & & \multicolumn{5}{|c|}{$n$} & \multicolumn{5}{|c|}{$n$} \\
\hline & & 6 & 10 & 20 & 30 & 50 & 6 & 10 & 20 & 30 & 50 \\
\hline \multirow{12}{*}{$C(\theta, \sigma)$} & $D_{1}$ & 167 & 150 & 211 & 264 & 397 & 284 & 310 & 463 & 552 & 693 \\
\hline & $D_{11}$ & 348 & 340 & 360 & 420 & 527 & 255 & 321 & 416 & 530 & 669 \\
\hline & $D_{2}$ & 348 & 321 & 338 & 359 & 472 & 444 & 453 & 533 & 602 & 738 \\
\hline & $D_{22}$ & 365 & 341 & 380 & 403 & 521 & 436 & 480 & 583 & 663 & 782 \\
\hline & $D_{3}$ & 271 & 223 & 287 & 320 & 448 & 280 & 281 & 407 & 492 & 634 \\
\hline & $D_{4}$ & 326 & 297 & 349 & 367 & 490 & 221 & 268 & 380 & 474 & 646 \\
\hline & $W W_{0}$ & 314 & 281 & 339 & 367 & 492 & 238 & 274 & 393 & 503 & 672 \\
\hline & $W W_{11}$ & 351 & 324 & 391 & 423 & 554 & 248 & 300 & 450 & 558 & 719 \\
\hline & $W W_{21}$ & 321 & 326 & 379 & 415 & 536 & 157 & 156 & 255 & 390 & 595 \\
\hline & $a a_{21}$ & 628 & 685 & 756 & 742 & 656 & 534 & 620 & 753 & 808 & 817 \\
\hline & $a a_{22}$ & 683 & 743 & 789 & 757 & 660 & 608 & 710 & 825 & 852 & 826 \\
\hline & $a a_{12}$ & 700 & 748 & 777 & 761 & 658 & 600 & 707 & 829 & 856 & 827 \\
\hline \multirow{12}{*}{$S(5)$} & $D_{1}$ & 98 & 175 & 430 & 702 & 980 & 213 & 356 & 690 & 879 & 990 \\
\hline & $D_{11}$ & 11 & 56 & 268 & 583 & 957 & 32 & 168 & 569 & 831 & 987 \\
\hline & $D_{2}$ & 95 & 163 & 756 & 999 & 1000 & 196 & 402 & 840 & 1000 & 1000 \\
\hline & $D_{22}$ & 39 & 57 & 267 & 861 & 1000 & 59 & 173 & 685 & 950 & 1000 \\
\hline & $D_{3}$ & 113 & 206 & 520 & 777 & 991 & 208 & 360 & 685 & 879 & 991 \\
\hline & $D_{4}$ & 71 & 164 & 820 & 1000 & 1000 & 205 & 441 & 868 & 1000 & 1000 \\
\hline & $W W_{0}$ & 81 & 186 & 770 & 1000 & 1000 & 207 & 435 & 865 & 1000 & 1000 \\
\hline & $W W_{11}$ & 11 & 44 & 304 & 869 & 1000 & 23 & 167 & 715 & 958 & 1000 \\
\hline & $W W_{21}$ & 32 & 64 & 307 & 860 & 1000 & 25 & 151 & 694 & 953 & 1000 \\
\hline & $a a_{21}$ & 59 & 83 & 294 & 960 & 1000 & 160 & 328 & 805 & 980 & 1000 \\
\hline & $a a_{22}$ & 32 & 50 & 208 & 899 & 1000 & 37 & 159 & 706 & 966 & 1000 \\
\hline & $a a_{12}$ & 20 & 31 & 132 & 638 & 1000 & 15 & 50 & 585 & 947 & 1000 \\
\hline
\end{tabular}


Al-Subh, S. A., Alodat, M. T., Ibrahim, K., \& Jemain, A. A. (2009). EDF goodness of fit tests of logistic distribution under selective order statistics. Pakistan Journal of Statistics, 25(3), 265-274.

Arnold, B. C., Balakrishnan, N., \& Nagaraja, H. N. (1992). A first course in order statistics. New York, NY: John Wiley and Sons.

Balakrishnan, N. (1992). Handbook of the logistic distribution. New York, NY: Marcel Dekker, Inc.

Bhoj, D. S. (1997). Estimation of parameters of the extreme value distribution using ranked set sampling. Communications in Statistics-Theory and Methods, 26(3), 653-667.

Chen, Z. (2000). On ranked-set sample quantiles and their applications. Journal of Statistical Planning and Inference, 83, 125-135.

D’Agostine, R., \& Stephens. M. (1986). Goodness of fit techniques. New York, NY: Marcel Dekker, Inc.

Dell, D. R., \& Clutter, J. L. (1972). Ranked set sampling theory with order statistics background. Biometrics, 28, 545-555.

Green J. R., \& Hegazy, Y. A. S. (1976). Powerful modified-EDF goodness-of-fit tests. Journal of the American Statistical Association, 71(353), 204-209.

Ibrahim, K. Alodat, M. T. Jemain, A. A., \& Al-Subh, S. A (2011). Chi-square test for goodness of fit for logistic distribution using ranked set sampling and simple random sampling. Statistica \& Applicazioni, IX(2), 111128.
McIntyre, G. A. (1952). A method of unbiased selective sampling using ranked sets. Australian Journal of Agricultural Research, 3, 385-390.

Muttlak, H. A. (1997). Median ranked set sampling. Journal of Applied Statistical Science, 6, 245-255.

Patil, G. P., Sinha, A. K., \& Taillie, C. (1994a). Ranked set sampling. In Handbook of statistics, 12: Environmental statistics, G.P. Patil \& C. R. Rao (Eds.), 167-200. New York: North Holland Elsevier.

Samawi, H. M., Ahmed, M. S., \& AbuDayyeh, W. (1996). Estimation the population mean using extreme ranked set sampling. Biometrical Journal, 38, 577-586.

Stephens, M. A. (1974). EDF statistics for goodness of fit and some comparisons. Journal of the American Statistical Association, 69, 730-737.

Stephens, M. A. (1979). Tests of fit for the logistic distribution based on the empirical distribution function. Biometrika, 66(3), 591595.

Stockes S. L., \& Sager T. W. (1988). Characterization of a ranked -set sample with application to estimating distribution functions. Journal of the American Statistical Association, 83(402), 374-381.

Takahasi, K., \& Wakitmoto, K. (1968). On unbiased estimates of the population mean based on the sample stratified by means of ordering. Annals of the Institute of Statistical Mathematics, 20, 1-31. 